\title{
ON RANDOM COINCIDENCE AND FIXED POINTS FOR A PAIR OF MULTIVALUED AND SINGLE-VALUED MAPPINGS
}

LJUBOMIR B. ĆIRIĆ, JEONG S. UME, AND SINIŠA N. JEŠIĆ

Received 2 February 2006; Revised 21 June 2006; Accepted 22 July 2006

Let $(X, d)$ be a Polish space, $\mathrm{CB}(X)$ the family of all nonempty closed and bounded subsets of $X$, and $(\Omega, \Sigma)$ a measurable space. A pair of a hybrid measurable mappings $f: \Omega \times X \rightarrow X$ and $T: \Omega \times X \rightarrow \mathrm{CB}(X)$, satisfying the inequality (1.2), are introduced and investigated. It is proved that if $X$ is complete, $T(\omega, \cdot), f(\omega, \cdot)$ are continuous for all $\omega \in \Omega, T(\cdot, x), f(\cdot, x)$ are measurable for all $x \in X$, and $f(\omega \times X)=X$ for each $\omega \in \Omega$, then there is a measurable mapping $\xi: \Omega \rightarrow X$ such that $f(\omega, \xi(w)) \in T(\omega, \xi(w))$ for all $\omega \in \Omega$. This result generalizes and extends the fixed point theorem of Papageorgiou (1984) and many classical fixed point theorems.

Copyright () 2006 Ljubomir B. Ćirić et al. This is an open access article distributed under the Creative Commons Attribution License, which permits unrestricted use, distribution, and reproduction in any medium, provided the original work is properly cited.

\section{Introduction and preliminaries}

Random fixed point theorems are stochastic generalizations of classical fixed point theorems. Random fixed point theorems for contraction mappings on separable complete metric spaces have been proved by several authors (Zhang and Huang [25], Hanš [6, 7], Itoh [8], Lin [12], Papageorgiou [13, 14], Shahzad and Hussian [19, 20], Špaček [22], and Tan and Yuan [23]). The stochastic version of the well known Schauder's fixed point theorem was proved by Sehgal and Singh [18].

Let $(X, d)$ be a metric space and $T: X \rightarrow X$ a mapping. The class of mappings $T$ satisfying the following contractive condition:

$$
\begin{aligned}
d(T x, T y) \leq & \alpha \max \left\{d(x, y), d(x, T x), d(y, T y), \frac{d(x, T y)+d(y, T x)}{2}\right\} \\
& +\beta \max \{d(x, T x), d(y, T y)\}+\gamma[d(x, T y)+d(y, T x)]
\end{aligned}
$$

for all $x, y \in X$, where $\alpha, \beta, \gamma$ are nonnegative real numbers such that $\beta>0, \gamma>0$, and $\alpha+$ $\beta+2 \gamma=1$, was introduced and investigated by Ćirić [1]. Ćirić proved that in a complete 
metric space such mappings have a unique fixed point. This class of mappings was further studied by many authors (Ćirić [2, 3], Singh and Mishra [21], and Rhoades et al. [16]). Singh and Mishra [21] have generalized Ćirić's [2] fixed point theorem to a common fixed point theorem of a pair of mappings and presented some application of such theorems to dynamic programming.

Let $(\Omega, \Sigma)$ be a measurable space with $\Sigma$ a sigma algebra of subsets of $\Omega$ and let $(X, d)$ be a metric space. We denote by $2^{X}$ the family of all subsets of $X$, by $\mathrm{CB}(X)$ the family of all nonempty closed and bounded subsets of $X$, and by $H$ the Hausdorff metric on $\mathrm{CB}(X)$, induced by the metric $d$. For any $x \in X$ and $A \subseteq X$, by $d(x, A)$ we denote the distance between $x$ and $A$, that is, $d(x, A)=\inf \{d(x, a): a \in A\}$.

A mapping $T: \Omega \rightarrow 2^{X}$ is called $\Sigma$-measurable if for any open subset $U$ of $X, T^{-1}(U)=$ $\{\omega: T(w) \cap U \neq \varnothing\} \in \Sigma$. In what follows, when we speak of measurability we will mean $\sum$-measurability. A mapping $f: \Omega \times X \rightarrow X$ is called a random operator if for any $x \in X$, $f(\cdot, x)$ is measurable. A mapping $T: \Omega \times X \rightarrow \mathrm{CB}(X)$ is called a multivalued random operator if for every $x \in X, T(\cdot, x)$ is measurable. A mapping $s: \Omega \rightarrow X$ is called a measurable selector of a measurable multifunction $T: \Omega \rightarrow 2^{X}$ if $s$ is measurable and $s(\omega) \in T(\omega)$ for all $\omega \in \Omega$. A measurable mapping $\xi: \Omega \rightarrow X$ is called a random fixed point of a random multifunction $T: \Omega \times X \rightarrow \mathrm{CB}(X)$ if $\xi(w) \in T(w, \xi(w))$ for every $w \in \Omega$. A measurable mapping $\xi: \Omega \rightarrow X$ is called a random coincidence of $T: \Omega \times X \rightarrow \mathrm{CB}(X)$ and $f: \Omega \times X \rightarrow X$ if $f(\omega, \xi(w)) \in T(w, \xi(w))$ for every $w \in \Omega$.

The aim of this paper is to prove a stochastic analog of the Ćirić [1] fixed point theorem for single-valued mappings, extended to a coincidence theorem for a pair of a random operator $f: \Omega \times X \rightarrow X$ and a multivalued random operator $T: \Omega \times X \rightarrow \mathrm{CB}(X)$, satisfying the following nonexpansive-type condition: for each $\omega \in \Omega$,

$$
\begin{aligned}
& H(T(\omega, x), T(\omega, y)) \\
& \leq \alpha(\omega) \max \{d(f(\omega, x), f(\omega, y)), d(f(\omega, x), T(\omega, x)), d(f(\omega, y), T(\omega, y)), \\
& \left.\quad\left(\frac{1}{2}\right)[d(f(\omega, x), T(\omega, y))+d(f(\omega, y), T(\omega, x))]\right\} \\
& +\beta(\omega) \max \{d(f(\omega, x), T(\omega, x)), d(f(\omega, y), T(\omega, y))\} \\
& +\gamma(\omega)[d(f(\omega, x), T(\omega, y))+d(f(\omega, y), T(\omega, x))]
\end{aligned}
$$

for every $x, y \in X$, where $\alpha, \beta, \gamma: \Omega \rightarrow[0,1)$ are measurable mappings such that for all $\omega \in \Omega$,

$$
\begin{gathered}
\beta(\omega)>0, \quad \gamma(\omega)>0, \\
\alpha(\omega)+\beta(\omega)+2 \gamma(\omega)=1 .
\end{gathered}
$$

\section{Main results}

Now we are proving our main result. 
Theorem 2.1. Let $(X, d)$ be a complete separable metric space, let $(\Omega, \Sigma)$ be a measurable space, and let $T: \Omega \times X \rightarrow \mathrm{CB}(X)$ and $f: \Omega \times X \rightarrow X$ be mappings such that

(i) $T(\omega, \cdot), f(\omega, \cdot)$ are continuous for all $\omega \in \Omega$,

(ii) $T(\cdot, x), f(\cdot, x)$ are measurable for all $x \in X$,

(iii) they satisfy (1.2), where $\alpha(\omega), \beta(\omega), \gamma(\omega): \Omega \rightarrow X$ satisfy (1.3) and (1.4).

If $f(\omega \times X)=X$ for each $\omega \in \Omega$, then there is a measurable mapping $\xi: \Omega \rightarrow X$ such that $f(\omega, \xi(w)) \in T(w, \xi(w))$ for all $\omega \in \Omega$ (i.e., $T$ and $f$ have a random coincidence point).

Proof. Let $\Psi=\{\xi: \Omega \rightarrow X\}$ be a family of measurable mappings. Define a function $g$ : $\Omega \times X \rightarrow R^{+}$as follows:

$$
g(\omega, x)=d(x, T(\omega, x))
$$

Since $x \rightarrow T(\omega, x)$ is continuous for all $\omega \in \Omega$, we conclude that $g(\omega, \cdot)$ is continuous for all $\omega \in \Omega$. Also, since $\omega \rightarrow T(\omega, x)$ is measurable for all $x \in X$, we conclude that $g(\cdot, x)$ is measurable (see Wagner [24, page 868]) for all $\omega \in \Omega$. Thus $g(\omega, x)$ is the Caratheodory function. Therefore, if $\xi: \Omega \rightarrow X$ is a measurable mapping, then $\omega \rightarrow g(\omega, \xi(w))$ is also measurable (see [17]).

Now we will construct a sequence of measurable mappings $\left\{\xi_{n}\right\}$ in $\Psi$ and a sequence $\left\{f\left(\omega, \xi_{n}(\omega)\right)\right\}$ in $X$ as follows. Let $\xi_{0} \in \Psi$ be arbitrary. Then the multifunction $G: \Omega \rightarrow$ $\mathrm{CB}(X)$ defined by $G(\omega)=T\left(w, \xi_{0}(w)\right)$ is measurable.

From the Kuratowski and Ryll-Nardzewski [11] selector theorem, there is a measurable selector $\mu_{1}: \Omega \rightarrow X$ such that $\mu_{1}(\omega) \in T\left(w, \xi_{0}(w)\right)$ for all $\omega \in \Omega$. Since $\mu_{1}(\omega) \in T\left(w, \xi_{0}(w)\right)$ $\subseteq X=f(\omega \times X)$, let $\xi_{1} \in \Psi$ be such that $f\left(\omega, \xi_{1}(\omega)\right)=\mu_{1}(\omega)$. Thus $f\left(\omega, \xi_{1}(\omega)\right) \in T(\omega$, $\left.\xi_{0}(\omega)\right)$ for all $\omega \in \Omega$.

Let $k: \Omega \rightarrow(1, \infty)$ be defined by

$$
k(\omega)=1+\frac{\beta(\omega) \gamma(\omega)}{2}
$$

for all $\omega \in \Omega$. Then $k(\omega)$ is measurable. Since $k(\omega)>1$ and $f\left(\omega, \xi_{1}(\omega)\right)$ is a selector of $T\left(w, \xi_{0}(w)\right)$, from Papageorgiou [13, Lemma 2.1] there is a measurable selector $\mu_{2}(\omega)=$ $f\left(\omega, \xi_{2}(\omega)\right) ; \xi_{2} \in \Psi$, such that for all $\omega \in \Omega$,

$$
\begin{gathered}
f\left(\omega, \xi_{2}(\omega)\right) \in T\left(\omega, \xi_{1}(\omega)\right), \\
d\left(f\left(\omega, \xi_{1}(\omega)\right), f\left(\omega, \xi_{2}(\omega)\right)\right) \leq k(\omega) H\left(T\left(\omega, \xi_{0}(\omega)\right), T\left(\omega, \xi_{1}(\omega)\right)\right) .
\end{gathered}
$$

Similarly, as $f\left(\omega, \xi_{2}(\omega)\right)$ is a selector of $T\left(w, \xi_{1}(w)\right)$, there is a measurable selector $\mu_{3}(\omega)=$ $f\left(\omega, \xi_{3}(\omega)\right)$ of $T\left(\omega, \xi_{2}(\omega)\right) \subseteq f(\omega \times X)$ such that

$$
d\left(f\left(\omega, \xi_{2}(\omega)\right), f\left(\omega, \xi_{3}(\omega)\right)\right) \leq k(\omega) H\left(T\left(\omega, \xi_{1}(\omega)\right), T\left(\omega, \xi_{2}(\omega)\right)\right) .
$$

Continuing this process we can construct a sequence of measurable mappings $\mu_{n}: \Omega \rightarrow X$, defined by $\mu_{n}(\omega)=f\left(\omega, \xi_{n}(\omega)\right) ; \xi_{n} \in \Psi$, such that

$$
\begin{gathered}
f\left(\omega, \xi_{n+1}(\omega)\right) \in T\left(\omega, \xi_{n}(\omega)\right) \\
d\left(f\left(\omega, \xi_{n}(\omega)\right), f\left(\omega, \xi_{n+1}(\omega)\right)\right) \leq k(\omega) H\left(T\left(\omega, \xi_{n-1}(\omega)\right), T\left(\omega, \xi_{n}(\omega)\right)\right) .
\end{gathered}
$$


4 On random coincidence and fixed points

Observe that condition (1.2) is clumsy. So, for simplicity, in the rest of the paper we will use this condition in the following form:

$$
\begin{aligned}
H(T(\omega, x), T(\omega, y)) \leq & \alpha(\omega) \max \left\{d(f(\omega, x), f(\omega, y)), \cdot, \cdot,\left(\frac{1}{2}\right)[\cdot+\cdot]\right\} \\
& +\beta(\omega) \max \{d(f(\omega, x), T(\omega, x)), d(f(\omega, y), T(\omega, y))\} \\
& +\gamma(\omega)[d(f(\omega, x), T(\omega, y))+d(f(\omega, y), T(\omega, x))] .
\end{aligned}
$$

From (2.7),

$$
\begin{aligned}
& H\left(T\left(\omega, \xi_{0}(\omega)\right), T\left(\omega, \xi_{1}(\omega)\right)\right) \\
& \leq \alpha(\omega) \max \left\{d\left(f\left(\omega, \xi_{0}(\omega)\right), f\left(\omega, \xi_{1}(\omega)\right)\right), \cdot, \cdot,\left(\frac{1}{2}\right)[\cdot+\cdot]\right\} \\
&+\beta(\omega) \max \left\{d\left(f\left(\omega, \xi_{0}(\omega)\right), T\left(\omega, \xi_{0}(\omega)\right)\right), d\left(f\left(\omega, \xi_{1}(\omega)\right), T\left(\omega, \xi_{1}(\omega)\right)\right)\right\} \\
&+\gamma(\omega)\left[d\left(f\left(\omega, \xi_{0}(\omega)\right), T\left(\omega, \xi_{1}(\omega)\right)\right)+d\left(f\left(\omega, \xi_{1}(\omega)\right), T\left(\omega, \xi_{0}(\omega)\right)\right)\right] .
\end{aligned}
$$

Since $f\left(\omega, \xi_{1}(\omega)\right) \in T\left(\omega, \xi_{0}(\omega)\right)$, then

$$
\begin{gathered}
d\left(f\left(\omega, \xi_{1}(\omega)\right), T\left(\omega, \xi_{0}(\omega)\right)\right)=0, \\
d\left(f\left(\omega, \xi_{0}(\omega)\right), T\left(\omega, \xi_{0}(\omega)\right)\right) \leq d\left(f\left(\omega, \xi_{0}(\omega)\right), f\left(\omega, \xi_{1}(\omega)\right)\right), \\
d\left(f\left(\omega, \xi_{1}(\omega)\right), T\left(\omega, \xi_{1}(\omega)\right)\right) \leq H\left(T\left(\omega, \xi_{0}(\omega)\right), T\left(\omega, \xi_{1}(\omega)\right)\right) .
\end{gathered}
$$

Thus from (2.8),

$$
\begin{aligned}
& H\left(T\left(\omega, \xi_{0}(\omega)\right), T\left(\omega, \xi_{1}(\omega)\right)\right) \\
& \leq \alpha(\omega) \max \left\{d\left(f\left(\omega, \xi_{0}(\omega)\right), f\left(\omega, \xi_{1}(\omega)\right)\right), \cdot, \cdot,\left(\frac{1}{2}\right)[\cdot+\cdot]\right\} \\
&+\beta(\omega) \max \left\{d\left(f\left(\omega, \xi_{0}(\omega)\right), f\left(\omega, \xi_{1}(\omega)\right)\right), H\left(T\left(\omega, \xi_{0}(\omega)\right), T\left(\omega, \xi_{1}(\omega)\right)\right)\right\} \\
&+\gamma(\omega)\left[d\left(f\left(\omega, \xi_{0}(\omega)\right), f\left(\omega, \xi_{1}(\omega)\right)\right)+H\left(T\left(\omega, \xi_{0}(\omega)\right), T\left(\omega, \xi_{1}(\omega)\right)\right)\right] .
\end{aligned}
$$

If we assume that $H\left(T\left(\omega, \xi_{0}(\omega)\right), T\left(\omega, \xi_{1}(\omega)\right)\right)>d\left(f\left(\omega, \xi_{0}(\omega)\right), f\left(\omega, \xi_{1}(\omega)\right)\right)$, then we have, as $\gamma(\omega)>0$,

$$
\begin{aligned}
& \gamma(\omega)[\left.d\left(f\left(\omega, \xi_{0}(\omega)\right), f\left(\omega, \xi_{1}(\omega)\right)\right)+H\left(T\left(\omega, \xi_{0}(\omega)\right), T\left(\omega, \xi_{1}(\omega)\right)\right)\right] \\
&<2 \gamma(\omega) H\left(T\left(\omega, \xi_{0}(\omega)\right), T\left(\omega, \xi_{1}(\omega)\right)\right) .
\end{aligned}
$$


Thus, from (1.4) and (2.10), we have

$$
\begin{aligned}
& H\left(T\left(\omega, \xi_{0}(\omega)\right), T\left(\omega, \xi_{1}(\omega)\right)\right) \\
&< \alpha(\omega) H\left(T\left(\omega, \xi_{0}(\omega)\right), T\left(\omega, \xi_{1}(\omega)\right)\right)+\beta(\omega) H\left(T\left(\omega, \xi_{0}(\omega)\right), T\left(\omega, \xi_{1}(\omega)\right)\right) \\
&+2 \gamma(\omega) H\left(T\left(\omega, \xi_{0}(\omega)\right), T\left(\omega, \xi_{1}(\omega)\right)\right) \\
&=(\alpha(\omega)+\beta(\omega)+2 \gamma(\omega)) H\left(T\left(\omega, \xi_{0}(\omega)\right), T\left(\omega, \xi_{1}(\omega)\right)\right) \\
&= H\left(T\left(\omega, \xi_{0}(\omega)\right), T\left(\omega, \xi_{1}(\omega)\right)\right),
\end{aligned}
$$

a contradiction. Therefore,

$$
H\left(T\left(\omega, \xi_{0}(\omega)\right), T\left(\omega, \xi_{1}(\omega)\right)\right) \leq d\left(f\left(\omega, \xi_{0}(\omega)\right), f\left(\omega, \xi_{1}(\omega)\right)\right) .
$$

Since $d\left(f\left(\omega, \xi_{1}(\omega)\right), T\left(\omega, \xi_{1}(\omega)\right)\right) \leq H\left(T\left(\omega, \xi_{0}(\omega)\right), T\left(\omega, \xi_{1}(\omega)\right)\right)$, we have

$$
d\left(f\left(\omega, \xi_{1}(\omega)\right), T\left(\omega, \xi_{1}(\omega)\right)\right) \leq d\left(f\left(\omega, \xi_{0}(\omega)\right), f\left(\omega, \xi_{1}(\omega)\right)\right) .
$$

By induction, we can show that

$$
\begin{gathered}
H\left(T\left(\omega, \xi_{n}(\omega)\right), T\left(\omega, \xi_{n+1}(\omega)\right)\right) \leq d\left(f\left(\omega, \xi_{n}(\omega)\right), f\left(\omega, \xi_{n+1}(\omega)\right)\right), \\
d\left(f\left(\omega, \xi_{n}(\omega)\right), T\left(\omega, \xi_{n}(\omega)\right)\right) \leq d\left(f\left(\omega, \xi_{n-1}(\omega)\right), f\left(\omega, \xi_{n}(\omega)\right)\right)
\end{gathered}
$$

for each $n \geq 1$ and all $\omega \in \Omega$. From (2.6) and (2.15),

$$
d\left(f\left(\omega, \xi_{n}(\omega)\right), f\left(\omega, \xi_{n+1}(\omega)\right)\right) \leq k(\omega) d\left(f\left(\omega, \xi_{n-1}(\omega)\right), f\left(\omega, \xi_{n}(\omega)\right)\right) .
$$

By (2.17), we get

$$
\begin{aligned}
d\left(f\left(\omega, \xi_{0}(\omega)\right), f\left(\omega, \xi_{2}(\omega)\right)\right) \leq & d\left(f\left(\omega, \xi_{0}(\omega)\right), f\left(\omega, \xi_{1}(\omega)\right)\right) \\
& +d\left(f\left(\omega, \xi_{1}(\omega)\right), f\left(\omega, \xi_{2}(\omega)\right)\right) \\
\leq & (1+k(\omega)) d\left(f\left(\omega, \xi_{0}(\omega)\right), f\left(\omega, \xi_{1}(\omega)\right)\right) .
\end{aligned}
$$

From (2.7),

$$
\begin{aligned}
& H\left(T\left(\omega, \xi_{0}(\omega)\right), T\left(\omega, \xi_{2}(\omega)\right)\right) \\
& \leq \alpha(\omega) \max \left\{d\left(f\left(\omega, \xi_{0}(\omega)\right), f\left(\omega, \xi_{2}(\omega)\right)\right), \cdot, \cdot,\left(\frac{1}{2}\right)[\cdot+\cdot]\right\} \\
&+\beta(\omega) \max \left\{d\left(f\left(\omega, \xi_{0}(\omega)\right), T\left(\omega, \xi_{0}(\omega)\right)\right), d\left(f\left(\omega, \xi_{2}(\omega)\right), T\left(\omega, \xi_{2}(\omega)\right)\right)\right\} \\
&+\gamma(\omega)\left[d\left(f\left(\omega, \xi_{0}(\omega)\right), T\left(\omega, \xi_{2}(\omega)\right)\right)+d\left(f\left(\omega, \xi_{2}(\omega)\right), T\left(\omega, \xi_{0}(\omega)\right)\right)\right] .
\end{aligned}
$$


Using (2.15), (2.16), (2.17), and (2.18) and the triangle inequality, we get

$$
\begin{aligned}
d\left(f\left(\omega, \xi_{2}(\omega)\right), T\left(\omega, \xi_{0}(\omega)\right)\right) \leq & \left.H\left(T\left(\omega, \xi_{1}(\omega)\right)\right), T\left(\omega, \xi_{0}(\omega)\right)\right) \\
\leq & d\left(f\left(\omega, \xi_{0}(\omega)\right), f\left(\omega, \xi_{1}(\omega)\right)\right), \\
d\left(f\left(\omega, \xi_{0}(\omega)\right), T\left(\omega, \xi_{2}(\omega)\right)\right) \leq & d\left(f\left(\omega, \xi_{0}(\omega)\right), f\left(\omega, \xi_{1}(\omega)\right)\right)+d\left(f\left(\omega, \xi_{1}(\omega)\right), f\left(\omega, \xi_{2}(\omega)\right)\right) \\
& +d\left(f\left(\omega, \xi_{2}(\omega)\right), T\left(\omega, \xi_{2}(\omega)\right)\right) \\
\leq & (1+k(\omega)) d\left(f\left(\omega, \xi_{0}(\omega)\right), f\left(\omega, \xi_{1}(\omega)\right)\right) \\
& +d\left(f\left(\omega, \xi_{1}(\omega)\right), f\left(\omega, \xi_{2}(\omega)\right)\right) \\
\leq & (1+2 k(\omega)) d\left(f\left(\omega, \xi_{0}(\omega)\right), f\left(\omega, \xi_{1}(\omega)\right)\right) .
\end{aligned}
$$

Now from (1.4), (2.17), (2.18), and (2.19), we have

$$
\begin{aligned}
& H\left(T\left(\omega, \xi_{0}(\omega)\right), T\left(\omega, \xi_{2}(\omega)\right)\right) \\
& \leq \alpha(\omega)(1+k(\omega)) d\left(f\left(\omega, \xi_{0}(\omega)\right), f\left(\omega, \xi_{1}(\omega)\right)\right) \\
&+\beta(\omega) k(\omega) d\left(f\left(\omega, \xi_{0}(\omega)\right), f\left(\omega, \xi_{1}(\omega)\right)\right) \\
&+2 \gamma(\omega)(1+k(\omega)) d\left(f\left(\omega, \xi_{0}(\omega)\right), f\left(\omega, \xi_{1}(\omega)\right)\right) \\
&= {[(1+k(\omega))(\alpha(\omega)+\beta(\omega)+2 \gamma(\omega))-\beta(\omega)] d\left(f\left(\omega, \xi_{0}(\omega)\right), f\left(\omega, \xi_{1}(\omega)\right)\right) } \\
&=(1+k(\omega)-\beta(\omega)) d\left(f\left(\omega, \xi_{0}(\omega)\right), f\left(\omega, \xi_{1}(\omega)\right)\right) .
\end{aligned}
$$

Hence we get, as $1+k(\omega)<2 k(\omega)$

$$
H\left(T\left(\omega, \xi_{0}(\omega)\right), T\left(\omega, \xi_{2}(\omega)\right)\right) \leq(2 k(\omega)-\beta(\omega)) d\left(f\left(\omega, \xi_{0}(\omega)\right), f\left(\omega, \xi_{1}(\omega)\right)\right)
$$

From (1.4) and (2.7) we have, as $f\left(\omega, \xi_{2}(\omega)\right) \in T\left(\omega, \xi_{1}(\omega)\right)$,

$$
\begin{aligned}
& H\left(T\left(\omega, \xi_{1}(\omega)\right), T\left(\omega, \xi_{2}(\omega)\right)\right) \\
& \leq \alpha(\omega) \max \left\{d\left(f\left(\omega, \xi_{1}(\omega)\right), f\left(\omega, \xi_{2}(\omega)\right)\right), \cdot, \cdot,\left(\frac{1}{2}\right)[\cdot+\cdot]\right\} \\
&+\beta(\omega) \max \left\{d\left(f\left(\omega, \xi_{1}(\omega)\right), T\left(\omega, \xi_{1}(\omega)\right)\right), d\left(f\left(\omega, \xi_{2}(\omega)\right), T\left(\omega, \xi_{2}(\omega)\right)\right)\right\} \\
&+\gamma(\omega) d\left(f\left(\omega, \xi_{1}(\omega)\right), T\left(\omega, \xi_{2}(\omega)\right)\right) .
\end{aligned}
$$

Since $f\left(\omega, \xi_{1}(\omega)\right) \in T\left(\omega, \xi_{0}(\omega)\right)$, by (2.23) we have

$$
\begin{aligned}
d\left(f\left(\omega, \xi_{1}(\omega)\right), T\left(\omega, \xi_{2}(\omega)\right)\right) & \leq H\left(T\left(\omega, \xi_{0}(\omega)\right), T\left(\omega, \xi_{2}(\omega)\right)\right) \\
& \leq(2 k(\omega)-\beta(\omega)) d\left(f\left(\omega, \xi_{0}(\omega)\right), f\left(\omega, \xi_{1}(\omega)\right)\right) .
\end{aligned}
$$


Thus from (2.17) and (2.24), we get

$$
\begin{aligned}
& H\left(T\left(\omega, \xi_{1}(\omega)\right), T\left(\omega, \xi_{2}(\omega)\right)\right) \\
& \leq \alpha(\omega) k(\omega) d\left(f\left(\omega, \xi_{0}(\omega)\right), f\left(\omega, \xi_{1}(\omega)\right)\right)+\beta(\omega) k(\omega) d\left(f\left(\omega, \xi_{0}(\omega)\right), f\left(\omega, \xi_{1}(\omega)\right)\right) \\
&+\gamma(\omega)(2 k(\omega)-\beta(\omega)) d\left(f\left(\omega, \xi_{0}(\omega)\right), f\left(\omega, \xi_{1}(\omega)\right)\right) \\
&= {[k(\omega)(\alpha(\omega)+\beta(\omega)+2 \gamma(\omega))-\beta(\omega) \gamma(\omega)] d\left(f\left(\omega, \xi_{0}(\omega)\right), f\left(\omega, \xi_{1}(\omega)\right)\right) . }
\end{aligned}
$$

Hence, as $\alpha(\omega)+\beta(\omega)+2 \gamma(\omega)=1$,

$$
H\left(T\left(\omega, \xi_{1}(\omega)\right), T\left(\omega, \xi_{2}(\omega)\right)\right) \leq(k(\omega)-\beta(\omega) \gamma(\omega)) d\left(f\left(\omega, \xi_{0}(\omega)\right), f\left(\omega, \xi_{1}(\omega)\right)\right)
$$

From (2.6) and (2.27),

$$
\begin{aligned}
d\left(f\left(\omega, \xi_{2}(\omega)\right), f\left(\omega, \xi_{3}(\omega)\right)\right) & \leq k(\omega) H\left(T\left(\omega, \xi_{1}(\omega)\right), T\left(\omega, \xi_{2}(\omega)\right)\right) \\
& \leq k(\omega)(k(\omega)-\beta(\omega) \gamma(\omega)) d\left(f\left(\omega, \xi_{0}(\omega)\right), f\left(\omega, \xi_{1}(\omega)\right)\right) .
\end{aligned}
$$

Since $k(\omega)=1+\beta(\omega) \gamma(\omega) / 2$, we have

$$
\begin{aligned}
k(\omega)(k(\omega)-\beta(\omega) \gamma(\omega)) & =\left(1+\frac{\beta(\omega) \gamma(\omega)}{2}\right)\left(1+\frac{\beta(\omega) \gamma(\omega)}{2}-\beta(\omega) \gamma(\omega)\right) \\
& =\left(1+\frac{\beta(\omega) \gamma(\omega)}{2}\right)\left(1-\frac{\beta(\omega) \gamma(\omega)}{2}\right) \\
& =1-\frac{\beta^{2}(\omega) \gamma^{2}(\omega)}{4} .
\end{aligned}
$$

Thus from (2.28),

$$
d\left(f\left(\omega, \xi_{2}(\omega)\right), f\left(\omega, \xi_{3}(\omega)\right)\right) \leq\left(1-\frac{\beta^{2}(\omega) \gamma^{2}(\omega)}{4}\right) d\left(f\left(\omega, \xi_{0}(\omega)\right), f\left(\omega, \xi_{1}(\omega)\right)\right)
$$

Analogously,

$$
d\left(f\left(\omega, \xi_{3}(\omega)\right), f\left(\omega, \xi_{4}(\omega)\right)\right) \leq\left(1-\beta^{2}(\omega) \gamma^{2}(\omega) / 4\right) d\left(f\left(\omega, \xi_{1}(\omega)\right), f\left(\omega, \xi_{2}(\omega)\right)\right)
$$

By induction,

$$
\begin{aligned}
d\left(f\left(\omega, \xi_{n}(\omega)\right), f\left(\omega, \xi_{n+1}(\omega)\right)\right) \\
\leq\left(1-\frac{\beta^{2}(\omega) \gamma^{2}(\omega)}{4}\right)^{[n / 2]} \\
\quad \times \max \left\{d\left(f\left(\omega, \xi_{0}(\omega)\right), f\left(\omega, \xi_{1}(\omega)\right)\right), d\left(f\left(\omega, \xi_{1}(\omega)\right), f\left(\omega, \xi_{2}(\omega)\right)\right)\right\},
\end{aligned}
$$


where $[n / 2]$ stands for the greatest integer not exceeding $n / 2$. Since $\beta(\omega) \gamma(\omega)>0$ for all $\omega \in \Omega$, from (2.32), we conclude that $\left\{f\left(\omega, \xi_{n}(\omega)\right)\right\}$ is a Cauchy sequence in $f(\omega \times X)$. Since $f(\omega \times X)=X$ is complete, there is a measurable mapping $f(\omega, \xi(\omega)) \in f(\omega \times X)$ such that

$$
\lim _{n \rightarrow \infty} f\left(\omega, \xi_{n}(\omega)\right)=f(\omega, \xi(\omega))
$$

Now by the triangle inequality and (1.2), we have

$$
\begin{aligned}
& d(f(\omega, \xi(\omega)), T(\omega, \xi(\omega))) \\
& \leq d\left(f(\omega, \xi(\omega)), f\left(\omega, \xi_{n+1}(\omega)\right)\right)+d\left(f\left(\omega, \xi_{n+1}(\omega)\right), T(\omega, \xi(\omega))\right) \\
& \leq d\left(f(\omega, \xi(\omega)), f\left(\omega, \xi_{n+1}(\omega)\right)\right)+H\left(T\left(\omega, \xi_{n}(\omega)\right), T(\omega, \xi(\omega))\right) \\
& \leq d\left(f(\omega, \xi(\omega)), f\left(\omega, \xi_{n+1}(\omega)\right)\right) \\
&+\alpha(\omega) \max \left\{d\left(f\left(\omega, \xi_{n}(\omega)\right), f(\omega, \xi(\omega))\right), \cdot, \cdot,\left(\frac{1}{2}\right)[\cdot+\cdot]\right\} \\
&+\beta(\omega) \max \left\{d\left(f\left(\omega, \xi_{n}(\omega)\right), T\left(\omega, \xi_{n}(\omega)\right)\right), d(f(\omega, \xi(\omega)), T(\omega, \xi(\omega)))\right\} \\
&+\gamma(\omega)\left[d\left(f\left(\omega, \xi_{n}(\omega)\right), T(\omega, \xi(\omega))\right)+d\left(f(\omega, \xi(\omega)), T\left(\omega, \xi_{n}(\omega)\right)\right)\right] .
\end{aligned}
$$

Thus

$$
\begin{aligned}
& d(f(\omega, \xi(\omega)), T(\omega, \xi(\omega))) \\
& \leq d\left(f(\omega, \xi(\omega)), f\left(\omega, \xi_{n+1}(\omega)\right)\right) \\
&+\alpha(\omega) \max \left\{d\left(f\left(\omega, \xi_{n}(\omega)\right), f(\omega, \xi(\omega))\right), \cdot, \cdot,\left(\frac{1}{2}\right)[\cdot+\cdot]\right\} \\
&+\beta(\omega) \max \left\{d\left(f\left(\omega, \xi_{n}(\omega)\right), f\left(\omega, \xi_{n+1}(\omega)\right)\right), d(f(\omega, \xi(\omega)), T(\omega, \xi(\omega)))\right\} \\
&+\gamma(\omega)\left[d\left(f\left(\omega, \xi_{n}(\omega)\right), T(\omega, \xi(\omega))\right)+d\left(f(\omega, \xi(\omega)), f\left(\omega, \xi_{n+1}(\omega)\right)\right)\right] .
\end{aligned}
$$

Taking the limit as $n \rightarrow \infty$, we get

$$
\begin{aligned}
d(f(\omega, \xi(\omega)), T(\omega, \xi(\omega))) \leq & \alpha(\omega) d(f(\omega, \xi(\omega)), T(\omega, \xi(\omega))) \\
& +\beta(\omega) d(f(\omega, \xi(\omega)), T(\omega, \xi(\omega))) \\
& +\gamma(\omega) d(f(\omega, \xi(\omega)), T(\omega, \xi(\omega))) \\
= & (1-\gamma(\omega)) d(f(\omega, \xi(\omega)), T(\omega, \xi(\omega))) .
\end{aligned}
$$

Hence $d(f(\omega, \xi(\omega)), T(\omega, \xi(\omega)))=0$, as $1-\gamma(\omega)<1$ for all $\omega \in \Omega$. Hence, as $T(\omega, \xi(\omega))$ is closed,

$$
f(\omega, \xi(\omega)) \in T(\omega, \xi(\omega)) \quad \forall \omega \in \Omega
$$


Remark 2.2. If in Theorem 2.1, $f(\omega, x)=x$ for all $(\omega, x) \in \Omega \times X$, then we get the following random fixed point theorem.

Corollary 2.3. Let $(X, d)$ be a separable complete metric space, let $(\Omega, \Sigma)$ be a measurable space, and let a mapping $T: \Omega \times X \rightarrow \mathrm{CB}(X)$ be such that $T(\omega, \cdot)$ is continuous for all $\omega \in \Omega, T(\cdot, x)$ is measurable for all $x \in X$, and

$$
\begin{aligned}
& H(T(\omega, x), T(\omega, y)) \\
& \quad \leq \alpha(\omega) \max \left\{d(x, y), d(x, T(\omega, x)), d(y, T(\omega, y)),\left(\frac{1}{2}\right)[d(x, T(\omega, y))+d(y, T(\omega, x))]\right\} \\
& \quad+\beta(\omega) \max \{d(x, T(\omega, x)), d(y, T(\omega, y))\}+\gamma(\omega)[d(x, T(\omega, y))+d(y, T(\omega, x))]
\end{aligned}
$$

for every $x, y \in X$, where $\alpha, \beta, \gamma: \Omega \rightarrow(0,1)$ are measurable mappings satisfying (1.2). Then there is a measurable mapping $\xi: \Omega \rightarrow X$ such that $\xi(w) \in T(w, \xi(w))$ for all $\omega \in \Omega$.

Corollary 2.4. Let $(X, d)$ be a complete separable metric space, let $(\Omega, \Sigma)$ be a measurable space, and let $f: \Omega \times X \rightarrow X$ and $T: \Omega \times X \rightarrow \mathrm{CB}(X)$ be two mappings satisfying the conditions (i) and (ii) in Theorem 2.1. If $f(\omega \times X)=X$ for each $\omega \in \Omega$ and $f$ and T satisfy the following condition:

$$
\begin{aligned}
& H(T(\omega, x), T(\omega, y)) \\
& \leq \lambda(\omega) \max \{d(f(\omega, x), f(\omega, y)), d(f(\omega, x), T(\omega, x)), d(f(\omega, y), T(\omega, y)), \\
& \left.\frac{d(f(\omega, x), T(\omega, y))+d(f(\omega, y), T(\omega, x))}{2}\right\},
\end{aligned}
$$

where $\lambda: \Omega \rightarrow(0,1)$ is a measurable function, then there is a measurable mapping $\xi: \Omega \rightarrow X$ such that $f(\omega, \xi(w)) \in T(w, \xi(w))$ for all $\omega \in \Omega$.

Proof. It is clear that if $f$ and $T$ satisfy (2.39), then $f$ and $T$ satisfy (1.2) with

$$
\alpha(\omega)=\lambda(\omega), \quad \beta(\omega)=\frac{1-\lambda(\omega)}{2}, \quad \gamma(\omega)=\frac{1-\lambda(\omega)}{4}
$$

Remark 2.5. If in Corollary 2.4, $f(\omega, x)=x$ for all $(\omega, x) \in \Omega \times X$, then we obtain the corresponding theorems of Hadžić [5] and Papageorgiou [13].

Finally, we give a simple example which shows that Theorem 2.1 and Corollaries 2.3 and 2.4 are actually an improvement of the results of Kubiak [10] and Papageorgiou [13]. Example 2.6. Let $(\Omega, \Sigma)$ be any measurable space and let $K=\{0,1,2,4,6\}$ be the subset of the real line. Let the mappings $f: \Omega \times K \rightarrow K$ and $T: \Omega \times K \rightarrow K$ be defined such that 
for each $\omega \in \Omega$,

$$
\begin{aligned}
& f(\omega, 0)=2, \quad f(\omega, 1)=4, \quad f(\omega, 2)=6, \quad f(\omega, 4)=0, \quad f(\omega, 6)=1, \\
& T(\omega, 0)=1, \quad T(\omega, 1)=2, \quad T(\omega, 2)=4, \quad T(\omega, 4)=0, \quad T(\omega, 6)=0 .
\end{aligned}
$$

Then $f$ and $T$ do not satisfy the contractive-type condition (2.39). Indeed, for $x=1$ and $y=2$, we have

$$
d(T(\omega, 1), T(\omega, 2))=2>\lambda(\omega) \max \left\{\|4-6\|,\|4-2\|,\|6-4\|, \frac{0+\|6-2\|}{2}\right\}=2 \lambda(\omega)
$$

for any $\lambda(\omega)<1$. On the other hand,

$$
d(T(\omega, 1), T(\omega, 2))=\frac{4}{5} \cdot 2+\frac{1}{10} \cdot 2+\frac{1}{20}(4+0) .
$$

Thus, for $x=1$ and $y=2, f$ and T satisfy (1.2) with $\alpha(\omega)=4 / 5, \beta(\omega)=1 / 10$, and $\gamma(\omega)=$ $1 / 20$. It is easy to show that $f$ and $T$ satisfy (1.2) for all $x, y \in K$, with the same $\alpha(\omega), \beta(\omega)$, and $\gamma(\omega)$. Also, the rest of assumptions of Theorem 2.1 is satisfied and for $\xi(\omega)=4$ we have

$$
f(\omega, \xi(\omega))=0=T(\omega, \xi(\omega))
$$

Note that $T$ does not satisfy (2.38) either, as for instance, for $x=0$ and $y=2$, we have

$$
\begin{aligned}
\alpha(\omega) \max & \left\{\|0-2\|,\|0-1\|,\|2-4\|, \frac{\|0-4\|+\|2-1\|}{2}\right\} \\
& +\beta(\omega) \max \{\|0-1\|,\|2-4\|\}+\gamma(\omega)[\|0-4\|+\|2-1\|] \\
= & \frac{5}{2} \alpha(\omega)+2 \beta(\omega)+5 \gamma(\omega)<3[\alpha(\omega)+\beta(\omega)+2 \gamma(\omega)]=3=d(T(\omega, 0), T(\omega, 2)) .
\end{aligned}
$$

Remark 2.7. Corollary 2.4 is a stochastic generalization and improvement of the corresponding fixed point theorems for contractive-type multivalued mappings of Ćirić [2], Ćirić and Ume [4], Kubiaczyk [9], Kubiak [10], Papageorgiou [14], and several other authors. Also Theorem 2.1 generalizes and extends the corresponding fixed point theorems for nonexpansive-type single-valued mappings of Ćirić [1] and Rhoades [15].

\section{Acknowledgment}

This research was financially supported by Changwon National University in 2006. 


\section{References}

[1] L. B. Ćirić, On some nonexpansive type mappings and fixed points, Indian Journal of Pure and Applied Mathematics 24 (1993), no. 3, 145-149.

[2] __ Nonexpansive type mappings and a fixed point theorem in convex metric spaces, Accademia Nazionale delle Scienze detta dei XL. Rendiconti. Serie V. Memorie di Matematica e Applicazioni. Parte I 19 (1995), 263-271.

[3] __ On some mappings in metric spaces and fixed points, Académie Royale de Belgique. Bulletin de la Classe des Sciences. 6e Série 6 (1995), no. 1-6, 81-89.

[4] L. B. Ćirić and J. S. Ume, Some common fixed point theorems for weakly compatible mappings, Journal of Mathematical Analysis and Applications 314 (2006), no. 2, 488-499.

[5] O. Hadžić, A random fixed point theorem for multivalued mappings of Ćirić's type, Matematički Vesnik 3(16)(31) (1979), no. 4, 397-401.

[6] O. Hanš, Reduzierende zufällige Transformationen, Czechoslovak Mathematical Journal 7 (1957), no. 82, 154-158.

[7] _ Random operator equations, Proceedings of the 4th Berkeley Symposium on Mathematical Statistics and Probability, vol. 2, part 1, University of California Press, California, 1961, pp. 185-202.

[8] S. Itoh, A random fixed point theorem for a multivalued contraction mapping, Pacific Journal of Mathematics 68 (1977), no. 1, 85-90.

[9] I. Kubiaczyk, Some fixed point theorems, Demonstratio Mathematica 9 (1976), no. 3, 507-515.

[10] T. Kubiak, Fixed point theorems for contractive type multivalued mappings, Mathematica Japonica 30 (1985), no. 1, 89-101.

[11] K. Kuratowski and C. Ryll-Nardzewski, A general theorem on selectors, Bulletin de l'Académie Polonaise des Sciences. Série des Sciences Mathématiques, Astronomiques et Physiques 13 (1965), 397-403.

[12] T.-C. Lin, Random approximations and random fixed point theorems for non-self-maps, Proceedings of the American Mathematical Society 103 (1988), no. 4, 1129-1135.

[13] N. S. Papageorgiou, Random fixed point theorems for multifunctions, Mathematica Japonica 29 (1984), no. 1, 93-106.

[14] _ Random fixed point theorems for measurable multifunctions in Banach spaces, Proceedings of the American Mathematical Society 97 (1986), no. 3, 507-514.

[15] B. E. Rhoades, A generalization of a fixed point theorem of Bogin, Mathematics Seminar Notes, Kobe University 6 (1978), no. 1, 1-7.

[16] B. E. Rhoades, S. L. Singh, and C. Kulshrestha, Coincidence theorems for some multivalued mappings, International Journal of Mathematics and Mathematical Sciences 7 (1984), no. 3, 429434.

[17] R. T. Rockafellar, Measurable dependence of convex sets and functions on parameters, Journal of Mathematical Analysis and Applications 28 (1969), no. 1, 4-25.

[18] V. M. Sehgal and S. P. Singh, On random approximations and a random fixed point theorem for set valued mappings, Proceedings of the American Mathematical Society 95 (1985), no. 1, 91-94.

[19] N. Shahzad and N. Hussain, Deterministic and random coincidence point results for $f$ nonexpansive maps, to appear in Journal of Mathematical Analysis and Applications.

[20] N. Shahzad and A. Latif, A random coincidence point theorem, Journal of Mathematical Analysis and Applications 245 (2000), no. 2, 633-638.

[21] S. L. Singh and S. N. Mishra, On a Ljubomir Ćirić's fixed point theorem for nonexpansive type maps with applications, Indian Journal of Pure and Applied Mathematics 33 (2002), no. 4, 531-542. 


\section{On random coincidence and fixed points}

[22] A. Špaček, Zufällige Gleichungen, Czechoslovak Mathematical Journal 5(80) (1955), no. 80, 462466.

[23] K.-K. Tan and X.-Z. Yuan, Random fixed-point theorems and approximation in cones, Journal of Mathematical Analysis and Applications 185 (1994), no. 2, 378-390.

[24] D. H. Wagner, Survey of measurable selection theorems, SIAM Journal on Control and Optimization 15 (1977), no. 5, 859-903.

[25] S. S. Zhang and N.-J. Huang, On the principle of randomization of fixed points for set-valued mappings with applications, Northeastern Mathematical Journal 7 (1991), no. 4, 486-491.

Ljubomir B. Ćirić: Faculty of Mechanical Engineering, University of Belgrade,

Aleksinačkih Rudara 12-35, Belgrade 11070, Serbia and Montenegro

E-mail address: 1ciric@afrodita.rcub.bg.ac.yu

Jeong S. Ume: Department of Applied Mathematics, Changwon National University,

Changwon 641-773, Korea

E-mail address: jsume@changwon.ac.kr

Siniša N. Ješić: Faculty of Electrical Engineering, University of Belgrade,

Bulevar Kralja Aleksandra 73, Belgrade 11000, Serbia and Montenegro

E-mail address: jesha@eunet.yu 